Minderheitsregierung

\section{Ein Lösungsansatz?}

Es wird viel über mögliche Koalitionen nach der Bundestagswahl spekuliert. Eine Annahme ist dabei immer, dass die neue Bundesregierung eine Mehrheit im Bundestag haben sollte. Wird dies tatsächlich unterstellt, dann ist nach den derzeitigen Umfrageergebnissen ${ }^{1}$ klar, dass jede der möglichen Koalitionen mindestens drei Parteien umfassen muss. Demnach wäre die SPD die stärkste Partei und hätte die Möglichkeit, eine Koalition zu bilden. Mögliche Koalitionen wären eine „Ampel“ mit SPD, Bündnis90/Die Grünen und FDP. Alternativ könnte die FDP durch die Partei Die Linke ersetzt werden. Nicht diskutiert wird hingegen eine „Kenia-Koalition“ aus SPD, Bündnis90/Die Grünen und der Union, auch weil diese Koalition offenbar nicht von der SPD gewünscht wird.

Die Dreierkonstellationen ohne Union haben jeweils zwei grundsätzliche Probleme, von denen eines bisher nicht diskutiert wird. Klar ist, dass SPD und Bündnis90/Die Grünen große Überschneidungen in den Politikfeldern haben und aktuell auch das Zusammengehen dieser beiden Parteien von den jeweiligen Repräsentant:innen befürwortet wird. Jeder der zur Auswahl stehenden beiden dritten möglichen Partner von SPD und Bündnis90/ Die Grünen weist aber so große inhaltliche Abweichungen auf, dass die für eine Koalition notwendige Kompromissfindung schwierig werden dürfte. So ist die FDP nicht nur strikt gegen die Steuererhöhungspläne von SPD und Bündnis90/Die Grünen, sondern sie sieht im Gegenteil sogar Entlastungen vor. Die Schwierigkeiten bei einer Koalition mit der Linkspartei liegen vor allem im Bereich der Außenpolitik, aber wohl auch bei der Einkommensteuer.

Unbeachtet ist bisher geblieben, dass keine dieser beiden Dreierkoalitionen im Bundesrat eine Mehrheit hätte. Bei im Bundesrat zustimmungspflichtigen Gesetzen, etwa zur Reform der Einkommensteuer, wird eine absolute Mehrheit an Ja-Stimmen benötigt (35 Stimmen). Enthaltungen gelten als Nein-Stimmen. Auch wird in den Koalitionsverträgen auf Landesebene oft festgelegt, dass im Falle der fehlenden Einigung der Koalitionäre Stimmenthaltung des Landes im Bundesrat geübt wird. Seit der kürzlich erfolgten Regierungsbildung in Sachsen-Anhalt vereini-

1 Dieser Kommentar ist vor der Bundestagswahl Online First auf der Website des Wirtschaftsdienst erschienen.

(C) Der/die Autor:in 2021. Open Access: Dieser Artikel wird unter der Creative Commons Namensnennung 4.0 International Lizenz veröffentlicht (creativecommons.org/licenses/by/4.0/deed.de).

Open Access wird durch die ZBW - Leibniz-Informationszentrum Wirtschaft gefördert. gen Landesregierungen, an denen die Union beteiligt ist, 51 Stimmen im Bundesrat aufeinander. Bei der SPD und bei Bündnis90/Die Grünen sind es 38 bzw. 41 Stimmen. Somit kann jede der drei Parteien ein im Bundesrat zustimmungspflichtiges Gesetz zum Scheitern bringen. Die FDP (18 Stimmen) und die Linkspartei (11 Stimmen) haben keine solche Mehrheit.

Was folgt nun aus dieser Konstellation? Werden wie in der Neuen Politischen Ökonomie üblich nutzenmaximierende Politiker:innen unterstellt, so bedeutet eine Dreier- gegenüber einer Zweierkoalition aus SPD und Bündnis90/Die Grünen, dass sie im Fall der Regierungsbildung Posten an einen dritten Koalitionspartner abgeben müssten. Auch müssten die knappen Haushaltsmittel eingesetzt werden, um die Klientel der dritten Partei zu bedienen. Das wiederum führt dazu, dass die eigene rot-grüne Klientel weniger Mittel erhält oder die Verschuldung bzw. die Steuern stärker erhöht werden müssten. Hinzu kommt, dass diese Nachteile nur mit geringen Vorteilen verbunden sind. Zwar erleichtert eine absolute Mehrheit im Bundestag das Regieren. Bei wichtigen Vorhaben aber, bei der auch die Mehrheit im Bundesrat benötigt wird, hätte keine der beiden derzeit diskutierten Dreierkoalitionen eine entsprechende Anzahl an Ja-Stimmen im Bundesrat. Nach einer Einigung innerhalb der Koalition müsste also immer noch die Union eingehegt werden. Vor dem Hintergrund der schwierigen Koalitionsbildung auf Bundesebene stellt sich die Frage, warum eine Bundesregierung zweimal versuchen soll, Kompromisse zu finden. Einmal müssten SPD und Bündnis90/Die Grünen mit der Linkspartei oder mit der FDP im Bereich der Einkommensteuer einen Kompromiss aushandeln. Dieser benötigt später aber zusätzlich noch die Zustimmung der Union.

Einfacher wäre es, wenn SPD und Bündnis90/Die Grünen gleich mit der Union einen Kompromiss suchen, ohne zusätzlich die FDP oder die Linkspartei einbinden zu müssen. Die Union will aber einer solchen Koalition nur beitreten, wenn Armin Laschet der Bundeskanzler wird. Nach den derzeitigen Umfrageergebnissen, wird dieser Fall aber nicht eintreten und diese Koalition nicht zustande kommen. Wenn schon ein offizielles Bündnis dieser Parteien nicht möglich ist, ein inoffizielles Bündnis aber für weitreichende Reformen notwendig ist, sollte auf einen dritten Partner in der Bundesregierung verzichtet werden. Die nächste Bundesregierung könnte also wenn das Wahlergebnis den derzeitigen Wahlumfragen entsprechen sollte - eine Minderheitsregierung aus SPD und Bündnis90/Die Grünen sein.

Michael Broer Ostfalia Hochschule für angewandte Wissenschaften m.broer@ostfalia.de 